\title{
INSIDE SOLARIS: THE PRESENCE OF GAME TECHNOLOGY IN ARCHITECTURAL DESIGN
}

\author{
SEAN PICKERSGILL \\ Louis Laybourne Smith School of Architecture and Design \\ University of South Australia \\ City West Campus, North Terrace, Adelaide, SA 5000, Australia \\ sean.pickersgill@unisa.edu.au
}

\section{GREG MORE}

Spatial Information Architecture Laboratory

Faculty of Architecture and Design

Royal Melbourne Institute of Technology

GPO Box 2476V, Melbourne, VIC 3001, Australia

gregory.more@rmit.edu.au

\begin{abstract}
Tarkovsky's 1971 film Solaris is an exquisitely evocative meditation on the relationship between technology, memory and loss - not only through it's presentation of the characters, narrative and themes of Stanislav Lem's novel, but also through the specific qualities of film as a medium of perception (Tarkovsky). Tarkovsky's oeuvre itself, particularly Nostalgia and Stalker, are tours-de-force in this respect. Yet there is always a melancholic distance between the viewer and the medium, perhaps impossible to traverse in any medium, but especially recognized in Tarkovsky's work. This in fact is a key issue, as Doležel has outlined in his study of literature and possible worlds, in the understanding of the force of fictional constructs within the representative arts, including literature (Doležel). The need to explain how it is possible and necessary to construct possible worlds that have both representative similarities with the real world and with hylomorphic qualities (i.e. a purposive and necessary form) in and of themselves is an opportunity that the making of digital environments cannot avoid.

This paper questions whether the same material for 'world-making' might be possible in persistent digital environments as there is in film. In the quest for an increasingly vivid experience of the presence of architecture, digital means of representation have achieved significant successes. Interestingly, there remains the opportunity to consider modes of digital realization as being sufficiently material within their mode of delivery - digital media. If we stay within the digital there are a number of modes by which its architectural product can be experienced. The question of this paper concerns the manner in which a recent mode of digital representation and exploration has developed: the game engine.
\end{abstract}




\section{Introduction}

Tarkovsky's 1971 film Solaris is an exquisitely evocative meditation on the relationship between technology, memory and loss - not only through it's presentation of the characters, narrative and themes of Stanislav Lem's novel, but also through the specific qualities of film as a medium of perception (Tarkovsky). Tarkovsky's oeuvre itself, particularly Nostalgia and Stalker, are tours-de-force in this respect. Yet there is always a melancholic distance between the viewer and the medium, perhaps impossible to traverse in any medium, but especially recognized in Tarkovsky's work. This in fact is a key issue as Doležel has outlined in his study of literature and possible worlds, in the understanding of the force of fictional constructs within the representative arts, including literature (Doležel). The need to explain how it is possible and necessary to construct possible worlds that have both representative similarities with the real world and with hylomorphic qualities (i.e. a purposive and necessary form) in and of themselves is an opportunity that the making of digital environments cannot avoid. In film there are distinctions between diagetic content - sounds, effects, music, points-of-view, that seem to follow intuitive aspects of the narrative, and non-diagetic elements - sounds, framing, music, events that draw attention to an origin external to the narratival space of the characters. For a film such as Solaris, the transformations of time, space and form that ensues in the narrative are defining aspects of the project itself, they are the key questions regarding the 'reality' of the story's events. This paper questions whether the same material for 'world-making' might be possible in persistent digital environments.

\section{Digital Issues in Architecture}

In the quest for an increasingly vivid experience of the presence of architecture, digital means of representation have achieved significant successes. It is a matter of record that processes made available by the computational strengths of parametric modeling and animation software has produced new models of the 'possible' in architecture. This mode of thinking remains enmeshed in the idea that modes of representation have the ultimate aim of being realized in a material form. Interestingly, there remains the opportunity to consider modes of digital realization as being sufficiently material within their mode of delivery - digital media. A more radical, but still robust, proposition might be that a mode of architecture that is principally delivered within the attenuated sensibilities of the digital might nevertheless be ontologically full in its apperception.

If we stay within the digital there are a number of modes by which its architectural product can be experienced. Within the limitations of the code of the applications, then the experience is limited to either a rendered view or an animation. These remain simulations of conventional modes of representation. The quality of the surfaces, the issue of the degree of simulation of the Real or its inherent abstraction, rehearse a conventional contest between mimetic practices. Whilst this can traverse work from the seamlessly natural of commercial digital artists to the selfconsciously contrived, for example Stephen Perrella's Hypersurfaces, the argument is familiar (Perrella). Whilst the subject matter of the representations may well be different, an empirical model opposed to an algorithmic process, the critical context is coeval.

A parallel issue, one that cannot be filly addressed in this paper, is the nature of the experience itself. Whether we consider the haptic content of AR and VR technology, pursued through the development of head-set, controller and other technology, or the optic issue of digital embodiment through forms of telepresence, the embodiment of the digital experience is considerably varied. For some, the nature of this question is pivotal as the search for seamless connectivity, whether by 'touching' the digital in some fashion or establishing developed emotional sensitivity to the avatar, is paramount. Even the relationship between avatars, the philosophical question of sentience and sapience in digital actors, is crucial. In all of these instances the appetite for total kinesthetic experience is intended to support a transition between normal bodily experience, a kind of empirical datum, and the incorporation of data sets that impart a spatialized experience. Instead, we would like to concentrate on the relationship between the representation of architecture, the expectations of vividness that may emerge from this process and the opportunity to see unique thematic experiences in digital environments that may emerge and contest our understanding of the 'real'.

\section{Game Engines}

The question of this paper concerns the manner in which a recent mode of digital representation and exploration has developed: the game engine. Game engines, as a form of code that traverses modeling, texturing, lighting and an animated scenographic view, rely on the workflow 
pipeline of 3D applications, but then place the content within first-person immersive environments. There are a number of questions that emerge from this process: How does this application differ, in architecture, from others in terms of its representative or mimetic role? how does it cater for issues of ontological vividness in comparison with AR and VR applications? how much use is it, for architecture, as a medium in comparison with the use of systemic, constructive algorithms?

Computer games consistently achieve levels of vividness that describe a position without precedent. In commercial games these spaces are influenced by narratological issues internal to specific tasks that are a part of a game, but in exploratory architectural work they are able to simultaneously act as a representation of a possible real and as a developed mise-en-scene of potential actions. They are both suffused with an aesthetic particular to the mechanics of the engine, how it delivers the idea of the Real, and the potential to act within this space. It is this last condition; the relentless need to consider the environments as persistent and transitive, which indicates the genuinely novel potential for digital environments. Put simply, if one could inhabit Piranesi's Carceri or Tarkovsky's Solaris, how would you act? These alternatives are qualitatively different, and mark a shift between architectural representation as an obsessive development of the architectural process of form-making, vs the spatialization of experience within the montage logic of film. The authors will present a description and analysis of work done within this media, arguing for its importance as a concept of 'the architectural' and hence within architectural education.

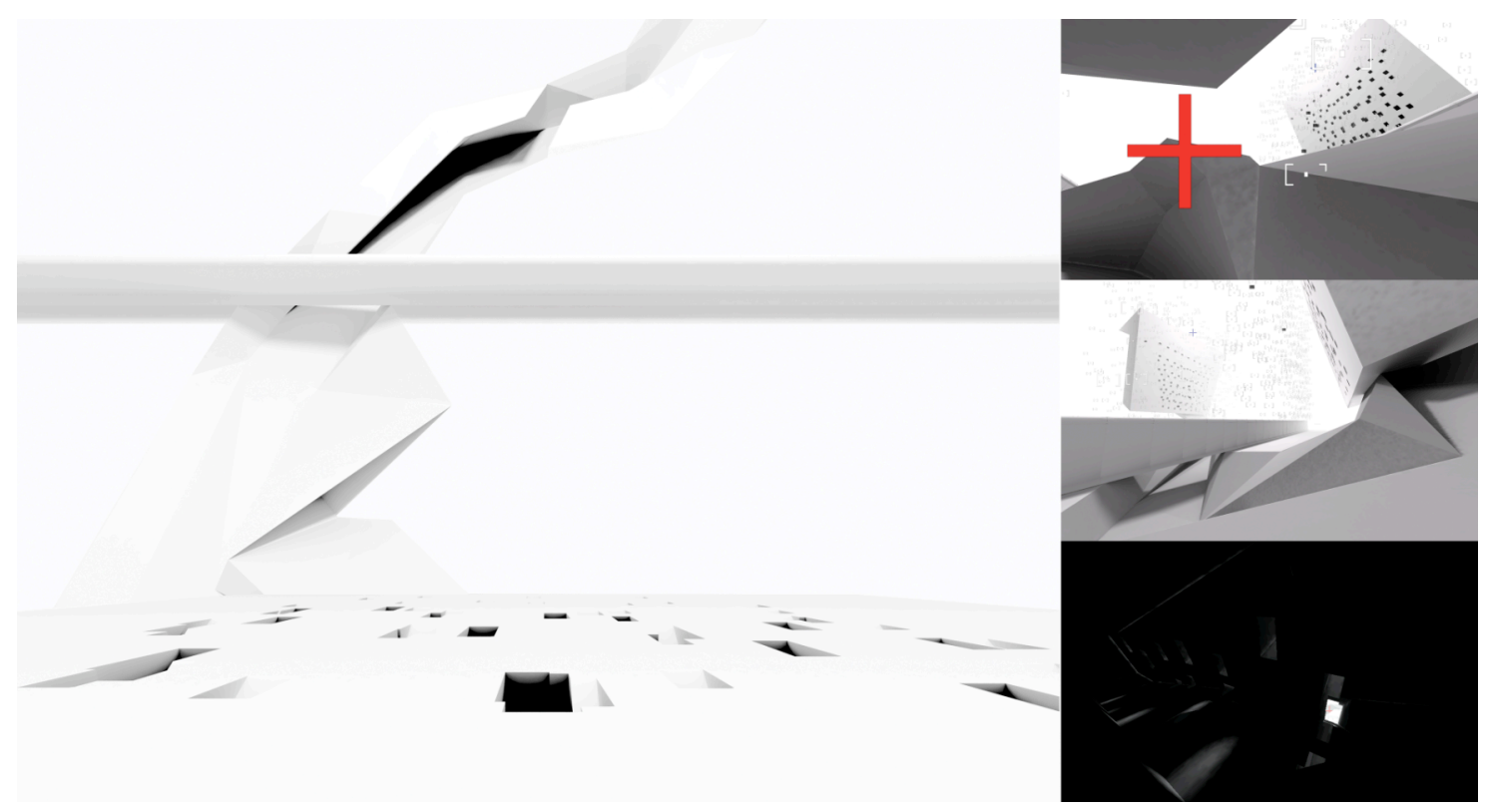

Figure 1: Greg More, Concrete Falls: 1000 Lines of Sight, 2006, screenshots

\section{Architecture and Representation}

There is nothing new in the idea that architectural design is driven by the means by which it is represented. A constant of architectural thinking is the manner in which the liminal aspects of architectural design, those aspects that seem to evade clear description, are governed by the search for the dissonant and unfamiliar. A governing presence in the evaluation of the oeuvre of practitioners is often the degree to which they pursued, or described a design agenda that is circumstantially different from the accepted sphere of influences, selected examples are: Eisenman's belligerent attempt to adopt structural linguistics into domesticity, Gehry's adoption of digital technologies as a mode of practice, ARM's adoption of a critical design practice that can simultaneously cherish and chide everyday culture and still find opportunities for revelation. In these instances there persists the accepted practice that the mode of presenting architecture is a valuable state of testing the relationship between the design and its reality.

Curiously, the critical apparatus of architectural criticism willingly considers aspects of unbuilt work in equal measure to that of built work. On empirical grounds this seems deficient, as there is clearly more material for investigation in a built work than is available in the product of 
representation. However, the counter argument is of course that it is through the means of representation that the most focused and semantically rich aspects of a work are presented. It's a challenging argument - that built work is only of marginal interest because of the confusing superfluity of its presence. Moreover, the extension of this argument seems to imply that meaning is only in use, not in intention - as if Ronchamp should always be considered in light of the cumulative vividness of pilgrims' experiences. In essence it is the same argument for a practice of thinking about the specificity of architecture that is being made again.

In an age in which the manner of representation is clearly evolving, the questions naturally resurface. Notwithstanding the structural similarities between the current means of representation and the history of the architectural image as a synechdocal effect of an overall design process, digital design involves the manipulation of a series of mimetic tools, themselves designed to reproduce the real as immaculately as possible - within certain parameters dictated by the physicality of the frustrum. Hence the significant subculture of practice dedicated to the reproduction of seamless or photo-real images. In architectural design, of course, the manner in which the play of the simulacra occurs is intimately linked with the manner in which the contract of authenticity is established between the design and its procession towards the actual. This is the commonsense view of the medium, in which its existence as a tool is premised in the real to be defined as a tool it must economically function as a device for perpetuating the economics of the same and simulation.

\section{$5 \quad$ Adamic Breeding - Things and Names}

However, the use of digital modeling software with the design mode involves a series of choices that superseded the mimetic function. By applying complex geometrical transformations to simple formal primitives, increasingly exotic formal entities can be created. Taken as a simple practice, this transformation of form can be considered as a mutation of topography (Cache) in which the idea of topography invokes a seamlessness of surface that destroys the notion of scale. Form, thence, is an infinite enfolding of matter to create a series of opportunistic spatialities, surfaces and abundances of program. The caesura between form and program is complete. Further, the re-naming of these instances (Lynn) as blobs, blebs, strands, etc invoked a new literacy in homophonic aspects of the voice of the digital. In both instances there is the Adamic encounter between the work and the idea of the typological - through a search for alternate modes of description, the monstrosities of the digital are positioned in some periodic table of formal motifs. Cache resisted this temptation and became forgotten as an innovator principally because of his resistance to incorporating the work of Objectile into a catalogue of formal characters (Cache). The novelty of the language is itself a complete strand of study, as it involves not only the determining of new nouns, but also adjectival and verbal practices that are incorporated into the design process. The term 'spline' is a pivotal case in point, in which the fashioning of polynomial lines and surfaces has become a key act of design surfacing.

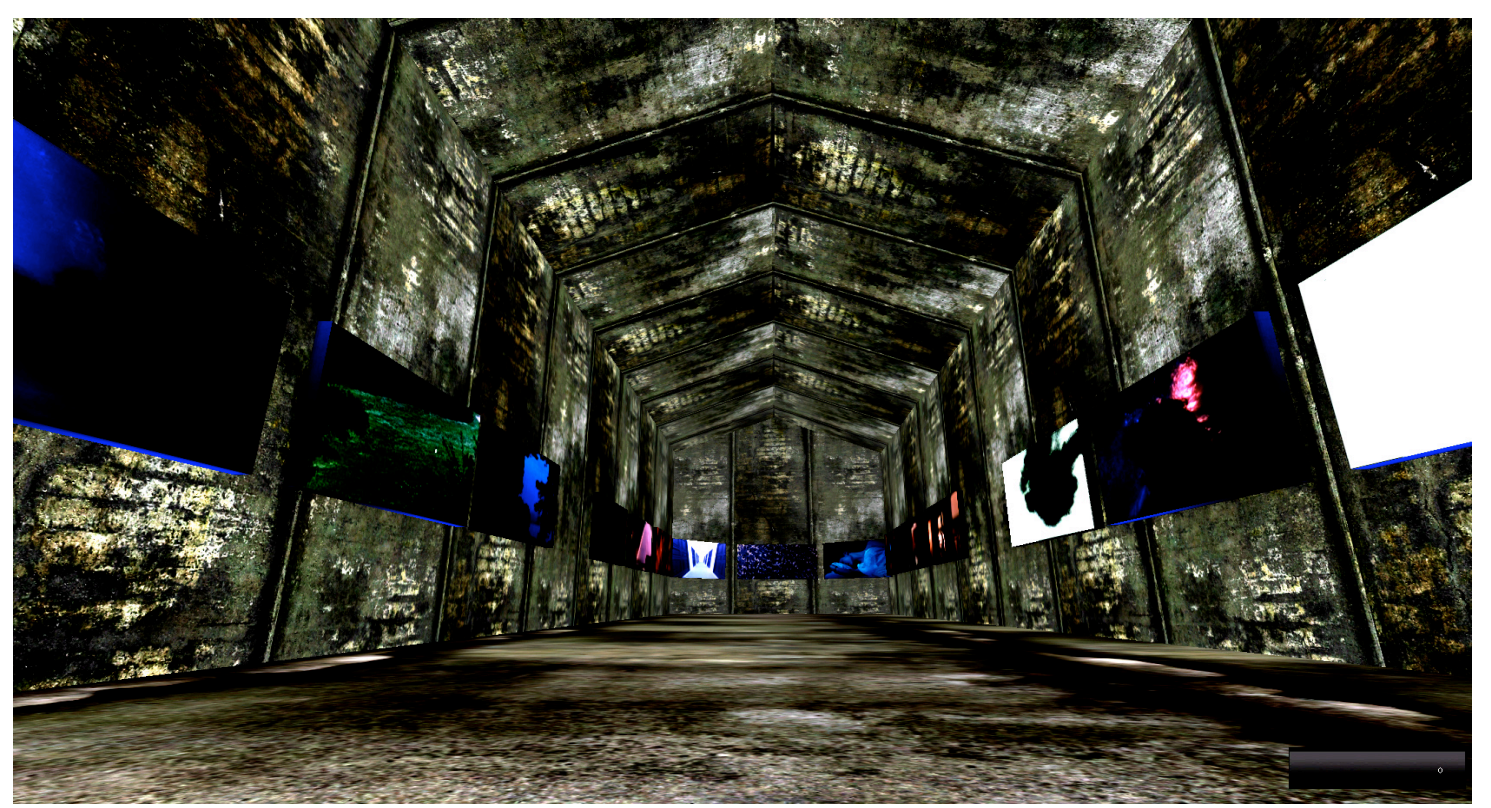

Figure 2: Sean Pickersgill, Bourgeois, 2006. screenshot 
Similarly, the borrowing of descriptions from biological organisms that have modes of selforganization for complex systems has been fused with an expanded definition of where the parameters of the architectural lie. It has become a meta-tecture, incorporating a dynamic isomorphic model that investigates where the act of design lies. In many instances this is replicating the radical empiricism of Deleuze (Logic, Deleuze). Again, though, the means by which this process is visualized is via the dynamic animation processes available within $3 d$ modeling software. Animation itself, as Lynn, has recognized, is a process of tweening that interpolates form between two or more static formal conditions, replicating in some instances the manner in which the eye will discretely adjust between the repetition of images produced at 24 or more frames per second. The differential is more though than just the convenience of allowing the animate to emerge from the dictates of the composed static. The differential preserves the opportunity for the new to emerge as an opportunity of form creation itself, as a model of how specific instances of the real make their presence in the world (Difference, Deleuze). Of course the application is premised on this process. In this instance the formal progeny that emerges once an epigenetic system is set in motion is made present by its means of representation. There is yet to be an alternative means by which epigenetically reiterative systems are made apprehensible and as such the claims that they represent an instance of a dynamic complex system are compromised. Whilst complex systems, of course, can be representatively 'shown' - the expanded analogy that they are inherently architectural needs more explanation (Fear).

In part this is pointed out by Manuel de Landa in his essay on Deleuze and the use of the genetic algorithm in architecture in which he points to the limitations in the use of algorithmic processes in architecture (De Landa). Implicitly, the transaction of 'explaining' the work, usually by referring to incommensurate data sets or the use of polynomial modifiers to visualize the morphological differences, tend to be descriptive of the process rather than explaining the manner in which 'this' form is appropriate. Indeed, in conversation, Bernard Cache referred to the selection as Kunstwollen, a will-to-select by some arbitrary sense of fitness. De Landa likens the process to that of a dog or horse breeder making choices about their animals (De Landa). In particular, the need to re-theorize the phenomenologically intimate is untouched, though Deleuze is clear on the need for the singularities of phenomenal experience to be treated as distinct entities, without them being subject to essentialist definitions (Difference, Deleuze).

So in architectural design, whether in practice or in its emulations within the design studio, the work of producing complex formal models requires the recoding of key terms within the language of architecture to imply a continuity of the project of the avant-garde. Thus, for example, Semper is invoked in discussing the use of surfaces, or strands, or the manipulation of UV texturing processes, whether the producing emerges from selective processes or not (Spuybroek). In many respects the arguments rehearsed the early twentieth century debate within the Deutsche Werkbund between Kunstwollen (a will to making form through the process of abstraction) and Typisierung (the delineation of typical models from which variations can emerge). We need only substitute the terms Objectile and Epigenetic for the debate to be contextualized to our present (Campbell).

\section{Hylomorphism - Unreal and The Real}

An alternate field in which an experience of form and programme takes place is that within persistent digital 'worlds'. These worlds are a consistent component of both stand-alone PC and console games that require a 3D environment for their games to occur within and MMOG spaces (Massive Multi-user Online Game) in which vast networked 3D communities exist. These take the form of both task-based (game) environments and social spaces. The most famous of the latter category, Second Life, is currently presenting itself as the evolution of digital communities made possible by the internet and associated technologies of mass communication. It allows users to exist as an avatar, of their own creation, within a 3D space that is dynamic in its evolution being constantly updated and modified by the participants. Both forms of these environments have formidable secondary communities devoted to social and economic aspects of the game/environment.

For no other reason, it would be sufficient for architecture to pay attention to these spaces given the fact that, for example, Second Life has property that participants construct digital spaces within. It would seem that the spatialised nature of the experience would lend itself to the translation of much of the theoretical digital work in architecture of the recent decade into this area. Yet this in itself is not enough to critically capitalize on the complex generative qualities of work such as Lynn and NOX, for example. This is a technological gap between the complexity of the models created within the digital architectural community and the level of complexity 
available to community environments such as Second Life. It would also neglect the clear intention for aspects of the architectural work to be made manifest in the real world - with all the complex sensate experiences that would entail.

But if we concentrate on the nature of the gamespace it is worth considering the manner in which the software necessary for creating a persistent environment is constructed. Game environment engines take polygonal models for both static and active elements of the map/level and apply the appropriate texture and lighting data to them in real time. The engine is able to optimise this process by only performing the texturing computations for those aspects of the model that are within the player's field of view. In addition, audio actors, environmental effects, and skyboxes all may be manipulated to increase the 'reality' of the experience.

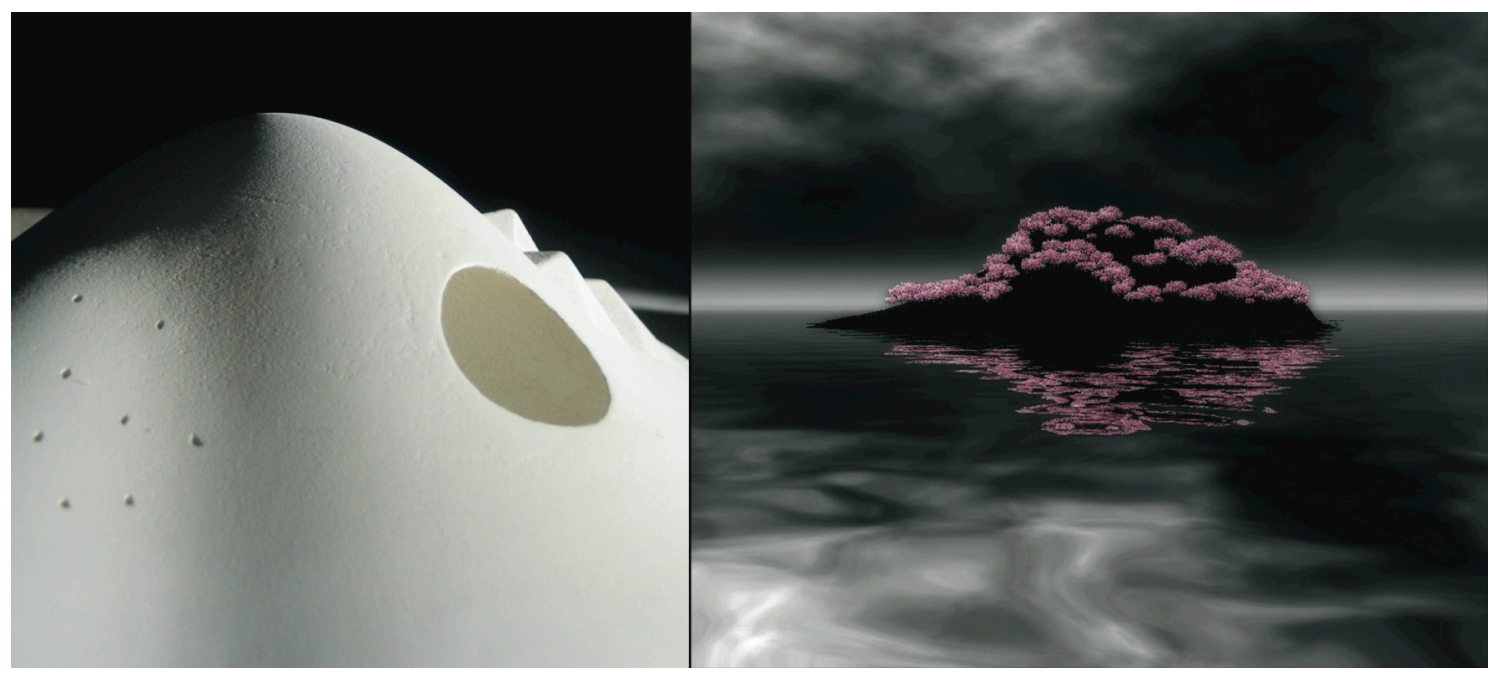

Figure 3: Greg More, Meta Island Beta, 2007, embedded sensor model and screenshot

Reality, in fact, is a key and contested aspect of the persistent digital environment. It is a matter of some debate within the game community as to the virtues of increased impressions of reality within games. Debate usually centres on a contest between narratological and ludological interests - are we there to experience something with textual depth or just to participate in some rule-based behaviour (Juul)? Debate usually follows the line that increased reality in representation is used to augment or cloud play (depending on which side of the debate one is on). I would suggest that a number of deeper philosophical questions are being enacted.

If you were to track the development of the representation of digital space over the last 25 years it is clear that key terms and questions recur that bear an uncanny resemblance to epistemological debates of the $17^{\text {th }}$ and $18^{\text {th }}$ century. The debate on the nature of vision and knowledge that originates in Descartes and Berkeley, particularly Berkeley's encomium esse est percipi (essence is perception) can be rehearsed within the constructed space of the digital environment (Berkeley). In some respects, like a clockwork universe, the answer is already known inasmuch as the programmers intuitively used these models in constructing a digital reality. Yet the texts of the philosophical works consistently approach the partitioning of experience into substances, attributes and geometrical metricisations that are mimicked by the evolution of game engine software.

What is interesting is, as digital environments grow to incorporate more complex forms of coded experiences, the degree to which 'knowing' an environment will be increasingly fraught perhaps productively so. Many of the issues that have emerged as questions for the mind/body dualism of pre-modern philosophies of logic and language are being enacted as we speak by the coding teams of Source and Epic Games. The challenge for architectural theory is to situate itself within this development.

A key application used in this respect is UnrealEd, a modeling application that accompanies the commercial FPS (First Person Shooter) game Unreal Tournament. Like all FPS environments, they are composed of forms that have their genesis in the same 3D modeling applications currently used by architecture. However, as indicated above, there are significant differences in the experience of the digital content. Unreal proposes a simulation of the real that is ontologically different from that of the conventional 3D modeling tool and its representations. 
The 3d tool exports it's sense as a static image, whereas Unreal paints a 'Berkeleyen' perspective idiosyncratically particular to the individual. Importantly too, it escapes the charge that it is merely solipsism because of the transaction of fictional vividness between programming team and player. It is in this sense that the idea of the digital world having pseudo-hylomorphic qualities is most appropriate. The FPS environment encourages a form of coded phenomenology, in which it is possible to entertain an engagement with simulacra as a 'genuinely' mediated quality itself. Much in the fashion that avant-garde music has investigate the sonic ambiences of aural effects of recording and reproduction as both a mimetic and collagistic practice.

\section{Trifurcation}

We suggest that this medium can develop in three directions: (i) towards an increasingly texturalised and reflexive practice that explores the qualia of the digital (un)real; (ii) towards an increasingly hyper-real attempt to emulate the immensely complex, and resource intensive, aspects of the artificial world; (iii) it may fetishize a nostalgia for low-fi models of itself as a means of recuperating control of the medium for independent agents with limited means. In essence the latter tow conditions involve the issue of labour and the ownership of the resources necessary to create these conditions. The last mode of practice, low-fi, is about the creation of digital environments sufficiently abstract to be performable and exportable to a broad community. Paradoxically, this practice is in some ways the most 'humanist' of the three as it assumes that the key interaction between the individual and the game environment lies in the ludic aspects, the 'play' of the individual as an emotive agent. The hyper-real environment, currently being pursued by the large game design and hardware manufacturing corporations is attempting to emulate a play experience that can deliver increasingly sophisticated morphological, textural, and physics-based effects. Without consciously recognizing it, and it is outside the scope of this discussion to do it justice, they are emulating the map/territory problem in philosophy - a problem that argues that no representation can be fully real and hence radical solipsism seems to be our endemic state.

The third opportunity, reflexive qualia, will investigate the manner in which the component elements of digital reality are scripted and reflexively recognize the experience of the digital. For architecture this may well incorporate not only an expanded knowledge of such issues as the map/territory dilemma mentioned above, or the question of whether there is possible empathy with the sentient and sapient characteristic of other avatars - the p-zombie problem, but also the materiality of the digital environment and its role in being the mise-en-scene of types of experience as yet unnamed.

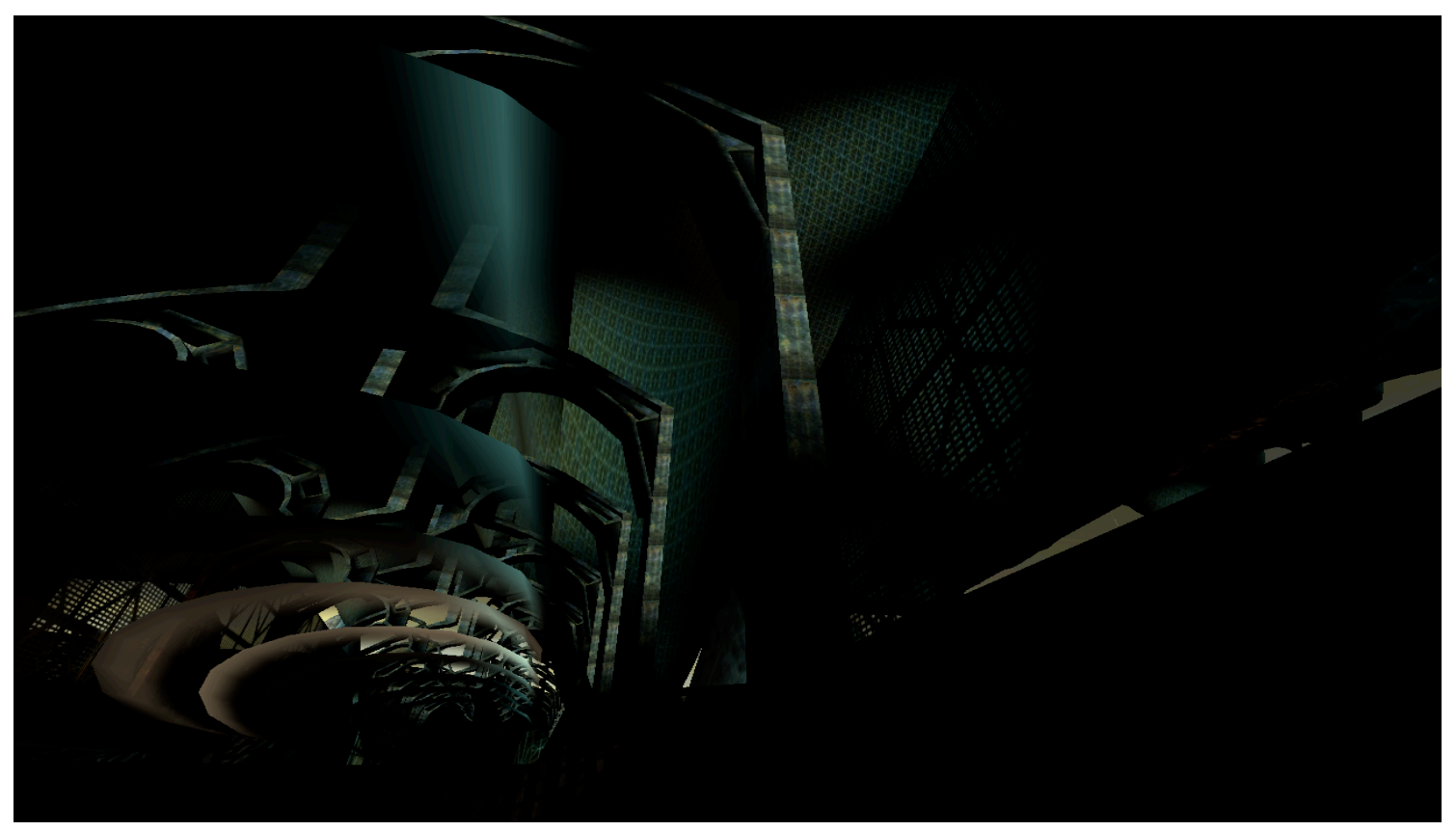

Figure 4: Sean Pickersgill, Terror-ain, 2006, screenshot 
Which returns us to the question of Tarkovsky and Solaris. If a task of architecture is to remember, remember the people that made it, remember the world that considered it new and vital, remember the promise of clarity and perspicacity in its processes, remember the vividness with which it mythologized itself and became the place in which imagination could occur, then the digital real has a task to fulfill. Digital environments, gamespaces, have the capability to offer an experience of the real that has both the frisson of mimetic fidelity - they look and sound real, whilst also permitting a vast array of counterintuitive events that may question the unfolding of experience. In a manner similar to the structural opportunities of film, both diagetic elements and non-diagetic elements can occur. Beyond the debate of the interrelationship between form and effect, and the sub-text of where the autonomous effects of design practice take place, the experientially full opportunity of the Unreal world is one place for future architecture. If we were to consider how one might build, code and explore the space station in Solaris, with its unnerving ability to make manifest the physical substance of our memories, what might we think of simulacra then?

\section{References}

Berkeley, George. A Treatise Concerning the Principles of Human Knowledge. Kessinger Press, 2004.

Cache, Bernard. Earthmoves, The Furnishing of Territories. MIT, 1995.

Campbell, Joan. The German Werkbund - The Politics of Reform in the Applied Arts. New Jersey: Princeton, 1978.

De Landa, Manuel. "Deleuze and the Use of the Genetic Algorithm in Architecture." in Rahim, Ali (ed.) Contemporary Techniques in Architecture. New York: Wiley, 2002.

Deleuze, Gilles. Difference and Repetition. Paris: Motilal Banarsidass, 2005.

Deleuze, Gilles. Logic of Sense. Columbia University Press, 1990.

Doležel, Lubomír. “Mimesis and Possible Worlds.” Poetics Today 9 (3) (1988): 475-496.

Doležel, Lubomír. Heterocosmica: Fiction and Possible Worlds. John Hopkins, 2000.

Fear, Bob (ed.), Architecture + Animation (Architectural Design). Academy Press, 2001.

Juul, Jesper. Half-Real. MIT Press, 2005.

Perella, Stephen (ed.). Hypersurface Architecture (Architectural Design Profile). John Wiley, 1998.

Spuybroek, Lars. Machining Architecture. Thames and Hudson, 2005.

Tarkovsky, Andrey, Sculpting in Time, Reflections on the Cinema. Austin: University of Texas Press, 1986. 